advised to activate the ECG recorder at the time of their symptoms. The ECG recordings were subsequently uploaded to a database and reviewed by a cardiologist. (Figure 1)

Results 39 patients (male 14, female 25, ages 13-86 years) were included. 38 patients submitted symptomatic recordings. A symptom-rhythm correlation was achieved in $37 / 38$ (in 1 case no ECG or symptom recording on return of device). Median time to diagnosis was 19 days (1-62). Diagnoses were: sinus rhythm 20 (54\%), sinus tachycardia 5 (13\%), ventricular ectopics 4 (11\%), SVT 6 (16\%), atrial fibrillation 2 (5\%) with time to diagnosis $16-40$ days.

Conclusion The MiCor device achieved a symptom-rhythm correlation in $97 \%$. In the majority of cases this permitted early reassurance and avoided further investigations. Where significant arrhythmia was diagnosed early implementation of therapy (including anticoagulation) was facilitated. The relative ease of use of this ECG recorder make it suitable for use in primary care where it may help reduce delays in diagnosis, improve patient experience, and reduce the number of secondary care referrals (table 1 ).

Conflict of Interest None

\section{PRE-PROCEDURE ANXIETY: THE ORIGIN OF PAIN DURING IMPLANTATION OF CARDIAC IMPLANTABLE ELECTRONIC DEVICES}

${ }^{1}$ Jonathan Senior*, 'Boyang Liu, ${ }^{2}$ Ayman Jani, 'Michail Apostolakis, ${ }^{3}$ Deepak Goyal, ${ }^{3}$ Robin Taylor, ${ }^{3}$ Will Foster, ${ }^{1}$ Nicola Brewster, ${ }^{3}$ David G Wilson. 'Worcester Royal Hospital; ${ }^{2}$ Birmingham Medical School; ${ }^{3}$ Worcestershire Acute Hospitals NHS Trust

\subsection{6/heartjnl-2019-BCS.38}

Introduction Cardiac implantable electronic device (CIED) implantation is an increasingly prevalent part of cardiac rhythm management. Adequate procedural pain management is central to the patient experience. The Association of Anaesthetists of Great Britain guidelines state that operators should develop techniques that permit the patient to undergo the procedure with minimum stress and maximum comfort. We developed an audit tool to assess the quality of pain management, and have evaluated the causative factors that can be targeted to optimise the patient experience.
Methods A prospective audit was collected over a 23-month period using a patient self-reporting questionnaire. Patients were asked to score their anxiety level prior to, and their pain during the procedure on a $0-10$ visual scale. Finally, patients were asked whether the pain matched their expectations.

Results 349 near consecutive patients $(67 \%$ male, age $75 \pm 13$ years) were included in this audit. There were 167 pacemaker implantations, 42 implantable cardioverter defibrillators, 98 cardiac resynchronisation devices, 13 lead revisions and 29 generator changes. A majority of patients $(219,63 \%)$ reported no/mild pain, with 172 (49\%) reporting the pain to be less than expected. However, 71 (21\%) patients reported the pain to be more than expected, with $56(16 \%)$ reporting severe pain.

We wanted to clarify which factors were important in influencing patients' experience of pain during their procedure. Using a logistic regression model, we investigated whether gender, weight, pre-implant anxiety, device complexity and operator predicted patients' experience of pain. Complex devices are defined as CRT, ICD, or any procedure involving lead extraction.

The logistic regression model was statistically significant $(\square 2$ 18.8, $\mathrm{P}=0.002$ ), explaining $14.8 \%$ (Nagelkerke $\mathrm{R} 2$ ) of the variance in severe pain and correctly classified $85.8 \%$ of cases. Patients with high pre-procedural worry were 2.7 times more likely to experience severe pain. Additionally, female patients were 3.2 times more likely to experience severe pain. Device complexity, operator and patient weight had no impact on pain (figure 1).

Interestingly, there was a weak positive correlation between the quantity of lidocaine usage and perceived pain $(\mathrm{R}=0.20$, $\mathrm{P}<0.001)$. It is possible that operators are using more local anaesthetic if patients are complaining of increased intra-procedural pain.

Conclusion The majority of patients underwent CIED implantation with minimum discomfort, though an important minority reported severe pain during the procedure. The most significant predictor of pain was a high level of pre-procedure anxiety and therefore interventions targeted at reducing preand peri-implant anxiety, particularly in women, warrant further investigation as a means to reduce pain during CIED implantation.

\begin{tabular}{llllllll}
\hline-5 & -3 & -1 & 1 & 3 & 5 & 7
\end{tabular}

Abstract 40 Figure 1 Forest plot demonstrating odds ratio $(95 \% \mathrm{Cl})$ derived from logistic regression for the prediction of severe pain 


\section{Conflict of Interest Nil}

\section{DOES PROGRESSIVE FIRST DEGREE AV BLOCK INDICATE THE NEED FOR PACING?}

Han Bin Xiao*, Waleed Arshad, Arvinder Kurbaan, Tim Bowker. Homerton University Hospital

\subsection{6/heartjnl-2019-BCS.39}

Background First-degree AV block, though considered benign, has been reported to be associated with an increased risk for atrial fibrillation, permanent pacing, heart failure and all-cause mortality. It is possible the extent of PR prolongation indicates the severity of adverse outcome. This study investigated whether the length of PR and its change with heart rate can predict the need for permanent pacing.

Method We studied 21 patients with a PR over $270 \mathrm{~ms}$. Nine patients underwent permanent pacing for symptomatic bradycardia or complete AV block and 12 did not. Two or more PR intervals and corresponding heart rates were measured. The unit change in PR to heart rate was calculated and defined as PR Index. We compared the two groups by Student $\mathrm{T}$ test or Fisher Chi Square test.

Result Age, sex distribution and baseline heart rate were similar in the two groups. The baseline mean PR interval was 70 ms greater (though did not reach statistical significance), a PR interval over $350 \mathrm{~ms}$ was more common (7/9 vs 3/12), the mean corrected PR interval by heart rate was significantly longer and the PR index was significantly higher in patients who were paced than in those who were not (all $\mathrm{p}<0.05)$.

Conclusion A very long PR interval may lead to AV dissociation requiring permanent pacing. A PR interval over $350 \mathrm{~ms}$ and an exaggerated prolongation of $\mathrm{PR}$ per given decrease in heart rate may indicate a high risk of $\mathrm{AV}$ dissociation, hence the need for permanent pacing.

Conflict of Interest None

\section{ATRIAL FIBRILLATION AND LONG-TERM SURVIVAL IN SOUTH ASIANS: INSIGHTS FROM THE UK ACALM REGISTRY}

${ }^{1}$ Rahul Potluri*, ${ }^{2}$ Kevin Bainey, ${ }^{3}$ Deepak Bhatt, ${ }^{4}$ Carlos Morillo, ${ }^{5}$ Billal Patel, ${ }^{5}$ Shajil Chalil, ${ }^{5}$ Ranjit More. ' ${ }^{1}$ lackpool Teaching Hospitals; ${ }^{2}$ Mazankowski Alberta Heart Institute; ${ }^{3}$ Harvard Medical School; ${ }^{4}$ Libin Cardiovascular Institute of Alberta; ${ }^{5}$ Blackpool Teaching Hospital NHS Foundation Trust

10.1136/heartjnl-2019-BCS.40

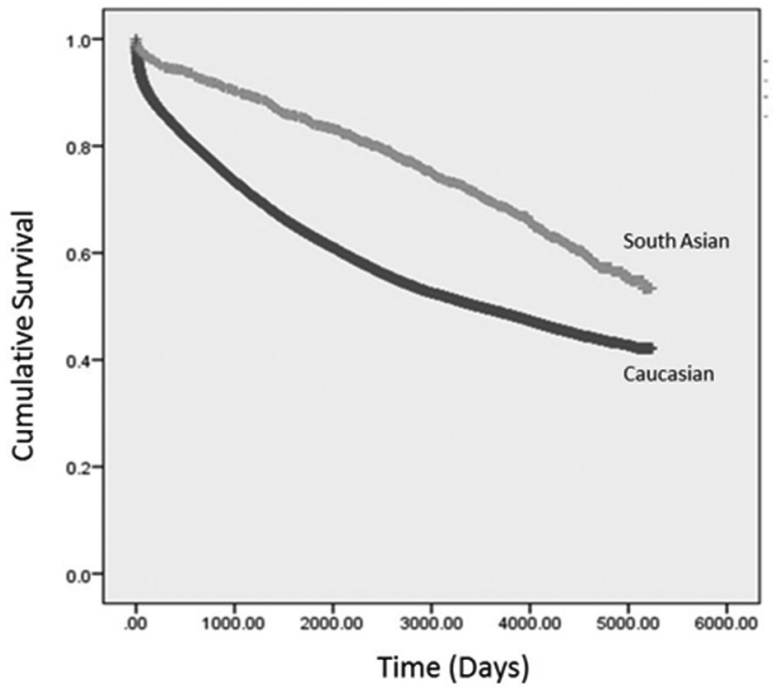

Abstract 42 Figure $1 \mathrm{KM}$ curve showing long term survival of Caucasian and South Asian AF patients

Background Atrial Fibrillation (AF) is the most common cardiac arrhythmia and is an independent risk factor for mortality. However, the impact of race has not been fully explored. We evaluated the frequency, clinical characteristics, and longterm survival in South Asian and Caucasian White patients with AF using a 'big data' approach.

Methods Using the United Kingdom (UK) Algorithm for Comorbidity, Associations, Length of stay, and Mortality (ACALM) registry, we analyzed 1,816,230 adults hospitalized between January 2000 and July 2014 using ICD-10 and OPCS-4 coding systems. Ethnic status was identified and AF patients were selected. Using logistic regression, we examined the odds of mortality adjusted for demographics and co-morbid status.

Results In our large cohort, South Asian patients had a lower frequency of $\mathrm{AF}$ hospitalization compared with Caucasian White patients $(0.8 \%$ [1876/243,363] versus 4.5\% [51,576/1, $151,222], p=<0.001)$. Differences in demographics and baseline characteristics are highlighted in the table below. KaplanMeier survival was worse in Caucasian White patients with AF (figure 1). Following adjustment, White race was associated with a higher long-term mortality compared with South Asian ethnicity in patients with AF (HR 1.38, 95\% CI 1.26-1.51, $\mathrm{p}<0.001$ ).

Conclusion In a 'big data' UK registry, AF hospitalization in South Asians appears less common and is associated with improved survival compared with Caucasian White patients.

\begin{tabular}{|l|c|c|c|}
\hline & South Asian (n=1876) & Caucasian White (n=51, 575) & $\mathrm{p}$-value \\
\hline Demographics & $65.5(14.3)$ & $74.2(12.2)$ & $<0.001$ \\
\hline Age (mean, SD) & 44.2 & 49.2 & $<0.001$ \\
\hline Female (\%) & 64.8 & 50.3 & $<0.001$ \\
\hline Selected baseline characteristics \\
\hline Hypertension (\%) & 43.2 & 17.3 & $<0.001$ \\
\hline Type 2 Diabetes (\%) & 10.7 & 7.2 & $<0.001$ \\
\hline Prior MI (\%) & 12.3 & 13.6 & 0.883 \\
\hline Prior heart failure (\%) & 3.5 & 8.9 & 0.04 \\
\hline Chronic kidney disease (\%) & $1.6(1.5)$ & $1.4(1.5)$ & $<0.001$ \\
\hline Charlson risk score & & & \\
\hline Clinical Outcome & 5.5 & 15.7 & $<0.001$ \\
\hline 1-year mortality \% & & &
\end{tabular}

